Physics

Physics Research Publications

\title{
Hartmann flow with braginsky viscosity: \\ A test problem for plasma in the intracluster medium
}

M. Lyutikov 
The Astrophysical Journal, 673:L115-L117, 2008 February 1

(C) 2008. The American Astronomical Society. All rights reserved. Printed in U.S.A.

\title{
HARTMANN FLOW WITH BRAGINSKY VISCOSITY: A TEST PROBLEM FOR PLASMA IN THE INTRACLUSTER MEDIUM
}

\author{
MAXIM LYUTIKOV \\ Department of Physics, Purdue University, 525 Northwestern Avenue, West Lafayette, IN 47907-2036 \\ Received 2007 September 25; accepted 2007 November 20; published 2008 January 9
}

\begin{abstract}
We consider a Hartmann layer, stationary flow of a viscose and resistive fluid between two plates with superimposed transverse magnetic field, in the limit of gyrotropic plasma, when viscosity across the field is strongly suppressed. For zero cross-field viscosity, the problem is not well posed, since viscosity then vanishes on the boundaries and in the middle of the layer, where there is no longitudinal field. An additional arbitrarily small isotropic viscosity allows one to find magnetic field and velocity profiles that are independent of this viscosity floor and different from flows with isotropic viscosity. Velocity sharply rises in a thin boundary layer, and the thinness of this boundary layer depends both on the Hartmann number and on the Lundquist number of the flow. The implication of the work is that, in simulating ICM dynamics, it is imperative to use numerical schemes that take into account anisotropic viscosity. Although magnetic fields are dynamically subdominant in the ICM, they do determine its dissipative properties, the stability of embedded structures, and the transition to turbulence.
\end{abstract}

Subject heading: galaxies: clusters: general

\section{INTRODUCTION}

Gasdynamical interactions of magnetized flows in the cores of clusters of galaxies play an important role in the formation of the observed morphological structures, e.g., by the expansion of an AGN-blown bubble into the intracluster medium (ICM) and the resulting plasma heating, and by the interaction of two gas components in merging clusters (e.g., Markevitch \& Vikhlinin 2007). The majority of theoretical work on these topics has been numerical, mostly using the existing fluid and MHD codes, like ZEUS. Unfortunately, for cluster cores, simple hydrodynamic models "face multiple failures" (Reynolds et al. 2005). Perhaps, the most evident examples are the Raleigh-Taylor and Kelvin-Helmholtz instabilities of AGN-blown bubbles; these instabilities disrupt the bubbles on approximately one rise time (e.g., Kaiser et al. 2005). On the other hand, artificial fiddling with viscosity - which is usually parameterized with respect to the Braginskii (1965) value (this procedure is not justified in the ICM; see Schekochihin et al. 2005 and Lyutikov 2007)—shows that "modest" changes of shear viscosity lead to qualitatively different results (increased viscosity makes the ICM plasma gellike and quenches the instability; see, e.g., Reynolds et al. 2005 and Sijacki \& Springel 2006).

One of principal reasons, perhaps, for the failure of simple MHD codes is that they use isotropic viscosity, whereas ICM plasma is strongly gyrotropic, in the sense that it is weakly collisional (i.e., $r_{\mathrm{L}} \ll \lambda \leq L$, where $r_{\mathrm{L}}$ is ion Larmor radius, $\lambda$ is mean free path, and $L$ is a typical size of the system) and weakly magnetized (i.e., the kinetic pressure $p$ is much larger than the magnetic pressure: $\beta=8 \pi p / B^{2} \gg 1$, where $B$ is a typical magnetic field). In ICM, the ion Larmor radius $\left(r_{\mathrm{L}} \sim\right.$ $\left.10^{8}-10^{9}\right) \mathrm{cm}$ ) is many orders of magnitude smaller than the mean free path $\left(\lambda \sim 10^{22}-10^{23} \mathrm{~cm}\right)$; the size $L \sim 10^{24} \mathrm{~cm}$, whereas $\beta \sim 100$ (Carilli \& Taylor 2002).

In a strongly gyrotropic plasma, the local transport properties, primarily viscosity and conductivity, become anisotropic (Braginskii 1965). The effects of anisotropic viscosity and conductivity are expected to substantially change the results of ICM simulations. As a simple example, note that the magnetic field is draped around the contact surface that separates the two interacting media (Lyutikov 2007). In a strongly gyrotropic plasma, the shear viscosity inside the draping layer, with a flow along magnetic field lines, then becomes 0 . This runs contrary to the idea that high viscosity provides stabilization (Reynolds et al. 2005). Note that draping itself can provide stabilization against Kelvin-Helmholtz instability, in a way that is similar to the effect that a thin layer of oil has on water (Dursi 2007).

Understanding the basic properties of strongly gyrotropic plasma is imperative for further progress, especially for parameterization of the "subgrid" physics in large numerical simulations. In this Letter, we adapt one of the basic solutions of MHD, the Hartmann flow (e.g., Landau \& Lifshitz 1984), to a viscosity that is anisotropic.

\section{HARTMANN FLOW WITH ANISOTROPIC VISCOSITY}

Let us consider a one-dimensional (along the $x$-direction) flow of weakly collisional plasma between two plates located at $z= \pm a$ with a superimposed external magnetic field (generally, oblique). This is meant to represent a boundary layer during the interaction of two plasma flows in the ICM. In the Chew-Goldberger-Low approximation (with zero Larmor radius and neglecting heat fluxes; Chew et al. 1956), the equations of resistive plasma flow read (Kulsrud 2005) as follows:

$$
\begin{gathered}
\frac{d \boldsymbol{v}}{d t}=-\nabla\left(P_{\perp}+B^{2} / 2\right)+\nabla\left[\hat{\boldsymbol{b}} \hat{\boldsymbol{b}}\left(P_{\|}-P_{\perp}-B^{2}\right)\right], \\
P_{\|}-P_{\perp}=3 \eta_{0} d_{t} \ln B=3 \eta_{0}(\hat{\boldsymbol{b}} \hat{\boldsymbol{b}} \nabla \boldsymbol{v}), \\
\partial_{t} \boldsymbol{B}=(\boldsymbol{\nabla} \times \boldsymbol{v}) \times \boldsymbol{B}+\eta_{r} \cdot \Delta \boldsymbol{B},
\end{gathered}
$$

where $\eta_{0}$ is the first Braginsky coefficient (Braginskii 1965), the resistivity $\eta_{r}$ is a tensor, and $\hat{\boldsymbol{b}}=\boldsymbol{B} / \boldsymbol{B}$ is a unit vector along the magnetic field. We also absorbed a factor $\sqrt{4 \pi}$ into the definition of the magnetic field.

Assuming that all quantities are independent of $x$ and $y$, from 


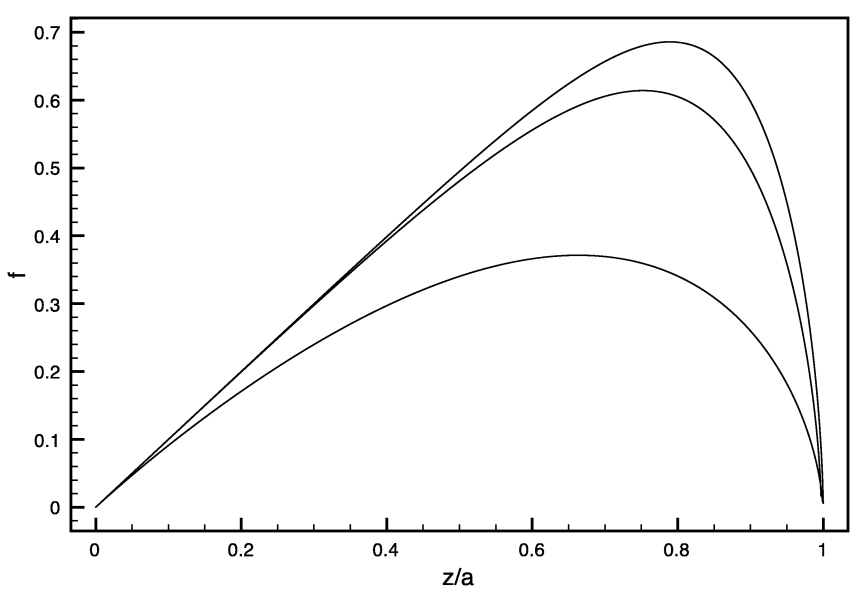

FIG. 1.-Function $f$ (longitudinal magnetic field) for $\alpha=1$ and different $G=1,3.1$, and 4.5 (from bottom to top) (larger values of $G$ are difficult to resolve numerically).

$\boldsymbol{\nabla} \cdot \boldsymbol{B}=0$, we find $B_{z}=$ const $=B_{0}$. Introducing $B_{x}=$ $B_{0} f(z), v_{x}=v$, the $x$-component of the Euler equation gives

$$
B_{0}^{2} f^{\prime}+3 \eta_{0} \partial_{z}\left[\frac{v^{\prime} f^{2}}{\left(1+f^{2}\right)^{2}}\right]-\partial_{x} P_{\perp}=0,
$$

where $\partial_{x} P_{\perp}=\Delta P / L$ is the constant pressure gradient driving the flow and $\Delta P$ is the drop in pressure over the length $L$. Equation (2) can be integrated once

$$
B_{0}^{2} f-\frac{\Delta P}{L}\left(z-z_{0}\right)+3 \eta_{0} \frac{v^{\prime} f^{2}}{\left(1+f^{2}\right)^{2}}=0 .
$$

The integration constant $z_{0}$ is a point where $f=0$.

Assuming that the $y$-component of the magnetic field vanishes, so that current flows across the magnetic field as $\boldsymbol{j}=$ $j_{y} e_{y}$, the resistivity equation gives

$$
v^{\prime}=-\eta_{\perp} f^{\prime \prime} .
$$

Here $\eta_{\perp}$ is the resistivity across the magnetic field. Finally, eliminating $v^{\prime}$ from equation (2) by use of equation (3), we get, for $f$, the following equation:

$$
B_{0}^{2} f-\frac{\Delta P}{L}\left(z-z_{0}\right)-3 \eta_{0} \eta_{\perp} \frac{f^{\prime \prime} f^{2}}{\left(1+f^{2}\right)^{2}}=0 .
$$

This is the main equation that determines the structure of the flow. For an anisotropic viscosity, it is a nonlinear equation. It differs from isotropic viscosity (e.g., Landau \& Lifshitz 1984 $\S 67$ ) by having a different viscosity term,

$$
\eta_{\mathrm{eff}}=3 \eta_{0} \frac{f^{2}}{\left(1+f^{2}\right)^{2}} .
$$

Renormalizing, $\quad z \rightarrow z a, \quad z_{0} \rightarrow z_{0} a$ and introducing $\alpha=$ $\left(B_{0}^{2} / \Delta P\right)(L / a)$, we find

$$
z_{0}-z+\alpha\left[f-\frac{1}{G^{2}} \frac{f^{\prime \prime} f^{2}}{\left(1+f^{2}\right)^{2}}\right]=0
$$

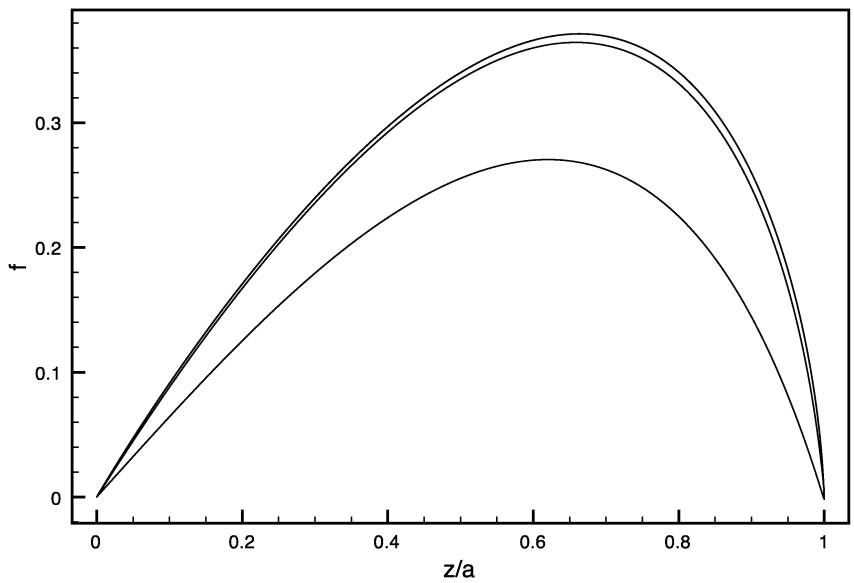

FIG. 2.-Effect of finite isotropic viscosity on the structure of magnetic field for $f_{r}=0.1,0.005,0.0001$, and $10^{-10}$ (from bottom to top), $\alpha=G=1$. For small enough $f_{r}$, solutions are nearly independent of the exact value of $f_{r}$.

where

$$
G=\frac{B_{0} a}{\sqrt{3 \eta_{0} \eta_{r}}}
$$

which we identify as the Hartmann number.

The boundary conditions are somewhat tricky in this case. From the continuity of the tangential magnetic field (assuming that there is no surface current) and from the symmetry of the flow, it is required that $f=f_{0}$ at $z= \pm a$ and that $f=0$ at point $z_{0}$, where $f_{0}$ is an imposed longitudinal magnetic field. Below we consider the case when there is no superimposed longitudinal magnetic field, $f_{0}=0$, and $z_{0}=0$.

On the other hand, at surfaces where $B_{x} \propto f=0$ (at the boundaries and in the middle), there is no viscosity (see eq. [6]), so the usual conditions of $v=0$ at $z= \pm a$ do not to be satisfied. Thus, in principle, the flow may slip along the boundaries and may have a discontinuity in the middle. This will make the problem unsolvable, as the order of the ordinary differential equation would be higher than the number of boundary conditions.

In fact, it is necessary to assume that there is some limit to the viscosity, to get a physically meaningful solution for equation (6), even if we just impose condition $f=0$ at $z= \pm a$. Near points where $f=0$, equation (6) reduces to $f=(z-$ $\left.z_{0}\right) / \alpha$, so that the derivative of $f$ at these points has a definite sign, given by the parameter $\alpha$. Thus, $f$ can be zero only once. And because $f=0$ in middle of the layer, it would clearly violate the conditions for a parallel magnetic field to vanish on the walls of the layer.

Introducing the resistivity limit $f_{r}$ in equation (6) by substituting $f^{2} \rightarrow f^{2}+f_{r}$, we can integrate equation (6) numerically (see Fig. 1).

For sufficiently small values of this viscosity limit, the final result is independent of its exact value (see Fig. 2).

The somewhat unphysical value of $\Delta P / L$ can be expressed in terms of the bulk velocity $v_{0}$ in the middle of the layer:

$$
\frac{\Delta P}{L} \sim \frac{v_{0} B_{0}^{2}}{G^{2} \eta_{\perp}}=\frac{v_{0} \eta_{0}}{a^{2}},
$$

which gives $\alpha=G^{2}\left(\eta_{\perp} / v_{0} a\right)=G^{2} / \mathrm{Lu}$, where we identified the $v_{0} a / \eta_{\perp}$ ratio with the magnetic Reynolds number Re or with 
the Lundquist number Lu. Parameter $\alpha$ plays an important role in determining the dynamics of the layer. If viscosity is dominated by ion-ion collisions, we can estimate $\alpha \sim 1 /\left(\beta M_{s} \mathrm{Kn}\right)$, where $\beta$ is the plasma $\beta$-parameter (the ratio of kinetic to magnetic pressure), $M=v_{0} / c_{s}$ is the flow Mach number (the ratio of velocity to sound speed $c_{s}$ ), and $\mathrm{Kn}=\lambda / a$ is the Knudsen number, which is the ratio of the mean free path $\lambda$ to the characteristic length scale $a$.

These solutions are quite different from those for isotropic viscosity (Landau \& Lifshitz 1984), and they have a number of particular features (Fig. 3). On the one hand, for large $G \gg 1$, in the bulk of the flow, the profile of $f$ is linearly increasing $(f \sim z / \alpha$; i.e., the flat velocity profile $)$, which is similar to that for isotropic viscosity. The parallel magnetic field drops back to 0 within a narrow boundary layer. Let us estimate the thickness of this boundary layer. Near the boundary, $f \rightarrow$ 0 , so that an approximate solution to equation (6) is

$$
f=\frac{z}{\alpha}-\frac{a}{\alpha}\left(\frac{z}{a}\right)^{\left(1+\sqrt{1+4 G^{2} \alpha^{2}}\right) / 2}
$$

If we define the thickness of the boundary layer $\delta$ when $f^{\prime}=0$, we find

$$
\frac{\delta}{a} \sim \frac{\ln (\alpha G)}{\alpha G} \sim \ln (\alpha G) \frac{\mathrm{Lu}}{G^{3}},
$$

where we assume $G \alpha \gg 1$. This expression can be compared with the that for isotropic viscosity, when $\delta_{i} / a \sim 1 / G$. The ratio $\delta / \delta_{i} \sim 1 / \alpha=\mathrm{Lu} / G^{2}$. Thus, parameter $\alpha=G^{2} /$ Lu measures the relative concentration of a magnetic field profile toward the wall; for $\alpha>1$, the boundary layer for anisotropic viscosity is narrower than that for isotropic viscosity.

In most applications, the $\mathrm{Lu} / G^{3}$ ratio is very small. For example, for the typical parameters of the ICM (i.e, velocity $\sim 1000 \mathrm{~km} \mathrm{~s}^{-1}$, layer thickness $\sim 1 \mathrm{kpc}$, and plasma $\beta=100$ ), we estimate $\mathrm{Lu}=10^{27}, G=10^{12}$, so that $\mathrm{Lu} / G^{3} \sim 10^{-9}$, and thus $\delta \ll a$.

\section{CONCLUSION}

In this Letter we considered a basic problem in plasma physics, i.e., the Hartmann flow with anisotropic viscosity. We first argued that, when transverse viscosity is suppressed completely, the problem cannot be formulated in a physically meaningful way; there should be some small isotropic contribution to viscosity. We derived magnetic field and velocity profiles, which, in the limit of small isotropic viscosity, are independent of its exact value. These profiles are considerably different from

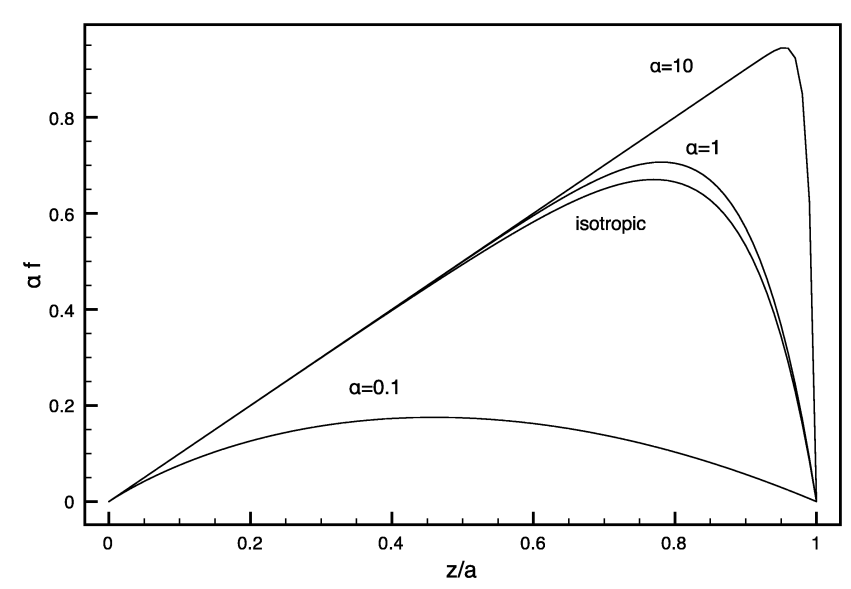

FIG. 3.-Comparison of analytical magnetic field profiles (multiplied by $\alpha$ for better graphical representation) for isotropic (Landau \& Lifshitz 1984; $f \propto[(z / a) \sinh G-\sinh (z G / a)] /(\cosh G-1))$ and approximate anisotropic (eq. [9]) viscosity for $G=10$ and different parameters $\alpha=0.1$ and 110. For $\alpha>1$, the boundary layer is narrower for anisotropic viscosity than it is for isotropic viscosity.

those for isotropic viscosity. The velocity gradients are much more concentrated close to the walls of the channel for anisotropic viscosity than they are for isotropic viscosity.

How important is the structure of a boundary layer for the overall structure of the flow? On the one hand, in a laminar regime at low Reynolds numbers, the structure of the boundary layer is probably not important; in the boundary layer. the relative velocity just drops to zero, according to some law, without affecting the overall structure of the flow. On the other hand, the properties of the boundary layer determine its stability and, thus, its transition to turbulence (Landau \& Lifshitz 1959). In ICM plasma, the Reynolds numbers are in the range Re 10-1000, while, typically, transition to turbulence occurs at $\operatorname{Re} \sim 100-1000$. (Transition to turbulence occurs in the boundary layer). Thus, we expect that the effects of anisotropic viscosity are likely to be very important for ICM plasma, especially for determining its transition to turbulence.

Thus, in simulating ICM dynamics, it is imperative that we use the appropriate numerical schemes that take into account anisotropic viscosity (and, to a lesser extent, conductivity), like the ones that have been applied to accretion disks (Sharma et al. 2007). Although magnetic fields are dynamically subdominant in the ICM (i.e., the plasma $\beta$-parameter is large), they do in fact determine its dissipative properties, the stability of embedded structures, and the transition to turbulence.

\section{REFERENCES}

Braginskii, S. I. 1965, Rev. Plasma Phys., 1, 205

Carilli, C. L., \& Taylor, G. B. 2002, ARA\&A, 40, 319

Chew, G. F., Goldberger, M. L., \& Low, F. E. 1956, Proc. R. Soc. London A, 236, 112

Dursi, L. J. 2007, ApJ, 670, 221

Kaiser, C. R., Pavlovski, G., Pope, E. C. D., \& Fangohr, H. 2005, MNRAS, 359,493

Kulsrud, R. M. 2005, Plasma Physics for Astrophysics (Princeton: Princeton Univ. Press)

Landau, L. D., \& Lifshitz, E. M. 1959, Fluid Mechanics (Oxford: Pergamon)
Landau, L. D., \& Lifshitz, E. M. 1984, Electrodynamics of Continuous Medium (Oxford: Pergamon)

Lyutikov, M. 2007, ApJ, 668, L1

Markevitch, M., \& Vikhlinin, A. 2007, Phys. Rep., 443, 1

Reynolds, C. S., McKernan, B., Fabian, A. C., Stone, J. M., \& Vernaleo, J. C. 2005, MNRAS, 357, 242

Schekochihin, A. A., Cowley, S. C., Kulsrud, R. M., Hammett, G. W., \& Sharma, P. 2005, ApJ, 629, 139

Sharma, P., Quataert, E., Hammett, G. W., \& Stone, J. M. 2007, ApJ, 667, 714

Sijacki, D., \& Springel, V. 2006, MNRAS, 371, 1025 